CRYSTALLOGRAPHIC COMMUNICATIONS

ISSN 2056-9890

Received 9 March 2018

Accepted 18 March 2018

Edited by A. J. Lough, University of Toronto, Canada

Keywords: crystal structure; organic co-crystal; hydrate; gallic acid; caffeine.

CCDC reference: 1830592

Supporting information: this article has supporting information at journals.iucr.org/e

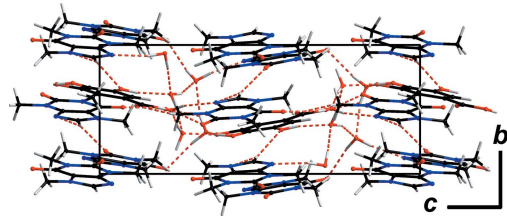

OPEN $\odot$ ACCESS

\section{Crystal water as the molecular glue for obtaining different co-crystal ratios: the case of gallic acid tris-caffeine hexahydrate}

\author{
L. Vella-Zarb and U. Baisch*
}

University of Malta, Msida, MSD 2080, Malta. *Correspondence e-mail: ulrich.baisch@um.edu.mt

The crystal structure of the hexahydrate co-crystal of gallic acid and caffeine, $\mathrm{C}_{7} \mathrm{H}_{6} \mathrm{O}_{5} \cdot 3 \mathrm{C}_{8} \mathrm{H}_{10} \mathrm{~N}_{4} \mathrm{O}_{2} \cdot 6 \mathrm{H}_{2} \mathrm{O}$ or GAL3CAF.6 $\mathrm{H}_{2} \mathbf{O}$, is a remarkable example of the importance of hydrate water acting as structural glue to facilitate the crystallization of two components of different stoichiometries and thus to compensate an imbalance of hydrogen-bond donors and acceptors. The water molecules provide the additional hydrogen bonds required to form a crystalline solid. Whereas the majority of hydrogen bonds forming the intermolecular network between gallic acid and caffeine are formed by crystal water, only one direct classical hydrogen bond between two molecules is formed between the carboxylic oxygen of gallic acid and the carbonyl oxygen of caffeine with $d(D \cdots A)=2.672(2) \AA$ A. All other hydrogen bonds either involve crystal water or utilize protonated carbon atoms as donors.

\section{Chemical context}

Gallic acid and its derivatives are widely known compounds in the pharmaceutical and chemical industry (Nayeem et al., 2016; Clarke et al., 2011). One such example is the dietary polyphenol found in Choerospondiatis fructus, a Mongolian medicinal herb used to treat conditions such as angina pectoris (Zhao et al., 2007). Lately, it has gained a lot of attention as a versatile component in crystal enineering, in particular with regards to co-crystallization and hydratation. Gallic acid could represent an entire microcosm of the special challenges and opportunities afforded by hydrates (Clarke et al., 2011) as it contains two of the most ubiquitous functional groups present in APIs: carboxylic acids and phenols. As part of a series of cocrystallization experiments in which both caffeine and gallic acid were used as coformers, single crystals of hydrated gallic acid and caffeine GAL3CAF. $6 \mathrm{H}_{2} \mathrm{O}$ in the ratio gallic acid:caffeine:water of 1:3:6 were obtained and characterized by single-crystal X-ray diffraction. The crystal structure is reported herein and compared to the different hydrated forms of this co-crystal GALCAF. $0.5 H_{2} O$ reported elsewhere (Clarke et al., 2010). The crystal structures differ greatly because of the different stoichiometry of the coformers. The different number of water molecules is necessary to act as structural glue, thereby facilitating crystallization.

\section{Structural commentary}

The asymmetric unit of the co-crystal GAL3CAF. $6 H_{2} \mathbf{O}$ consists of three independent caffeine molecules and one 
gallic acid molecule as well as six hydrate water molecules. Gallic acid can be described as a thrice-substituted benzoic acid with hydroxyl groups in both the meta and para positons. Caffeine consists of a purine backbone with carbonyl substituents at positions 2 and 6 (C26, C28, C46, C48, C66, C68) and methyl groups connected to three out of four nitrogen atoms (Fig. 1).<smiles>O=C(O)c1cc(O)c(O)c(O)c1</smiles>

\section{Gallic Acid}<smiles>Cn1c(=O)c2c(ncn2C)n(C)c1=O</smiles>

Caffeine
Bond distances of the aromatic rings and substituents of both types of molecules lie within the expected ranges and exhibit the usual lengths for aromatic, double or single homoor heteroatomic bonds. Only one nitrogen atom on each of the three caffeine molecules can act as a hydrogen-bond acceptor (N23, N43, N63) with the protonated carbon in the fivemembered ring (C22, C42, C62) acting as a weak hydrogenbond donor. Every molecule exhibits weak intramolecular interactions (Steiner, 2002). Whereas gallic acid forms intermoleclar bonds between two adjacent hydroxyl substituents $[\mathrm{O} 11-\mathrm{H} 11 \cdots \mathrm{O} 10, d(D \cdots A)=2.709(2) \AA]$, the caffeine molecules form weak interactions between two of three methyl carbons and two carbonyl oxygen or backbone nitrogen atoms, namely C31, C33, C51, C53, C71, C73 as well as O32, N43, O54, O72, and O74. Distances range between 2.71 and $2.95 \AA$. In comparison, the corresponding intramolecular hydrogen-bonding interactions in the published GALCAF $0.5 \mathrm{H}_{2} \mathrm{O}$ structure reported in the Cambridge Structural Database (Groom et al., 2016) with code MUPNOB (Clarke et al., 2010) have distances of $d(D \cdots \mathrm{A})_{\text {gallic acid }}=$ 2.743 (2), $2.712(2) \AA$ and $d(D \cdots A)_{\text {caffeine }}$ of $2.78-2.71 \AA$.

\section{Supramolecular features}

As a result of the limited number of hydrogen-bond donors and acceptors in both gallic acid and caffeine, the packing strongly depends on (i) the concentration of each of the components in solution as well as (ii) other experimental conditions such as other components in solution, temperature, pressure, etc. In fact, there is a large difference in the way both molecules pack in the crystal lattice.

The crystal structure of GALCAF. $0.5 \mathbf{H}_{2} \mathbf{O}$ (Clarke et al., 2010) has a 1:1:0.5 ratio of gallic acid, caffeine and water molecules. Both molecules form hydrogen-bonded tapes that are built by $\mathrm{COO}-\mathrm{H} \cdots \mathrm{N}$ and $\mathrm{O}-\mathrm{H} \cdots \mathrm{O}$ interactions $[\mathrm{O} \cdots \mathrm{N}$ $=2.705$ (2) $\AA$ and $\mathrm{O} \cdots \mathrm{O}=2.703$ (2) and 2.750 (2) $\mathrm{]}$ ] formed between the hydoxyl substituents on gallic acid molecules and the carbonyl moieties of adjacent caffeine molecules. These tapes are then cross-linked by water molecules that hydrogenbond with the third hydroxyl group in each gallic acid mol-
Table 1

Hydrogen-bond geometry $\left(\AA{ }^{\circ}\right)$.

\begin{tabular}{|c|c|c|c|c|}
\hline$D-\mathrm{H} \cdots A$ & $D-\mathrm{H}$ & $\mathrm{H} \cdots A$ & $D \cdots A$ & $D-\mathrm{H} \cdots A$ \\
\hline $\mathrm{O} 1-\mathrm{H} 1 \cdots \mathrm{O} 32^{\mathrm{i}}$ & $0.85(3)$ & $1.86(3)$ & $2.672(2)$ & $161(3)$ \\
\hline $\mathrm{O} 10-\mathrm{H} 10 \cdots \mathrm{O} 104$ & $0.86(4)$ & $1.79(4)$ & $2.643(2)$ & $174(4)$ \\
\hline $\mathrm{O} 11-\mathrm{H} 11 \cdots \mathrm{O} 10$ & $0.83(3)$ & $2.32(4)$ & $2.709(2)$ & $109(3)$ \\
\hline $\mathrm{O} 11-\mathrm{H} 11 \cdots \mathrm{O} 102$ & $0.83(4)$ & $1.88(4)$ & $2.680(2)$ & $161(3)$ \\
\hline $\mathrm{O} 12-\mathrm{H} 12 \cdots \mathrm{O} 100^{\mathrm{i}}$ & $0.86(3)$ & $1.86(3)$ & $2.702(2)$ & $166(3)$ \\
\hline $\mathrm{O} 100-\mathrm{H} 10 C \cdots \mathrm{O} 105$ & $0.83(4)$ & $2.07(4)$ & $2.851(3)$ & $157(4)$ \\
\hline $\mathrm{O} 100-\mathrm{H} 10 D \cdots \mathrm{O} 10$ & $0.82(4)$ & $2.74(3)$ & $3.286(2)$ & $126(3)$ \\
\hline $\mathrm{O} 100-\mathrm{H} 10 D \cdots \mathrm{O} 102$ & $0.82(4)$ & $2.02(4)$ & $2.798(3)$ & $158(3)$ \\
\hline $\mathrm{O} 101-\mathrm{H} 10 I \cdots \mathrm{N} 43^{\mathrm{ii}}$ & $0.94(4)$ & $1.97(4)$ & $2.898(3)$ & $170(3)$ \\
\hline $\mathrm{O} 101-\mathrm{H} 10 J \ldots \mathrm{O} 100$ & $0.88(4)$ & $2.09(4)$ & $2.960(3)$ & $170(4)$ \\
\hline $\mathrm{O} 102-\mathrm{H} 10 A \cdots \mathrm{O} 101^{\mathrm{iii}}$ & $0.89(4)$ & $1.87(4)$ & $2.754(3)$ & $169(4)$ \\
\hline $\mathrm{O} 102-\mathrm{H} 10 B \cdots \mathrm{O} 103$ & $0.85(4)$ & $1.85(4)$ & $2.691(3)$ & $173(3)$ \\
\hline $\mathrm{O} 103-\mathrm{H} 10 K \cdots \mathrm{O} 34^{\mathrm{iv}}$ & $0.78(5)$ & $2.06(5)$ & $2.832(3)$ & $168(5)$ \\
\hline $\mathrm{O} 103-\mathrm{H} 10 L \cdots \mathrm{N} 23^{\mathrm{v}}$ & $0.84(5)$ & $1.99(5)$ & $2.823(3)$ & $168(4)$ \\
\hline $\mathrm{O} 104-\mathrm{H} 10 G \cdots \mathrm{O} 1^{\mathrm{vi}}$ & $0.91(3)$ & $2.50(3)$ & $3.011(2)$ & $116(2)$ \\
\hline $\mathrm{O} 104-\mathrm{H} 10 G \cdots \mathrm{O} 105$ & $0.91(3)$ & $2.03(3)$ & $2.857(3)$ & $151(3)$ \\
\hline $\mathrm{O} 104-\mathrm{H} 10 H \cdots \mathrm{O} 74^{\mathrm{vi}}$ & $0.93(5)$ & $2.00(5)$ & $2.924(2)$ & $171(4)$ \\
\hline $\mathrm{O} 105-\mathrm{H} 10 E \cdots \mathrm{O} 74^{\mathrm{ii}}$ & $0.86(5)$ & $2.11(5)$ & $2.952(2)$ & $169(4)$ \\
\hline $\mathrm{O} 105-\mathrm{H} 10 F \cdots \mathrm{N} 63$ & $0.88(4)$ & $1.92(4)$ & $2.798(2)$ & $173(4)$ \\
\hline $\mathrm{C} 22-\mathrm{H} 22 \cdots \mathrm{O} 72^{\mathrm{vii}}$ & $0.97(3)$ & $2.38(3)$ & $3.227(3)$ & $146(2)$ \\
\hline $\mathrm{C} 30-\mathrm{H} 30 \mathrm{C} \cdots \mathrm{O} 12^{\mathrm{vii}}$ & 0.98 & 2.71 & 3.247 (3) & 115 \\
\hline $\mathrm{C} 31-\mathrm{H} 31 C \cdots \mathrm{O} 2^{\mathrm{vi}}$ & 0.98 & 2.40 & $3.326(3)$ & 158 \\
\hline $\mathrm{C} 42-\mathrm{H} 42 \cdots \mathrm{O} 12^{\text {viii }}$ & $0.92(3)$ & $2.45(3)$ & $3.254(3)$ & $146(3)$ \\
\hline $\mathrm{C} 42-\mathrm{H} 42 \cdots \mathrm{O} 72$ & $0.92(3)$ & $2.66(3)$ & $3.136(3)$ & $113(2)$ \\
\hline $\mathrm{C} 62-\mathrm{H} 62 \cdots \mathrm{O} 52^{\mathrm{vii}}$ & $0.96(3)$ & $2.24(3)$ & 3.119 (3) & $151(3)$ \\
\hline
\end{tabular}

Symmetry codes: (i) $x,-y+1, z-\frac{1}{2}$; (ii) $x,-y+2, z+\frac{1}{2}$; (iii) $x, y-1, z$; (iv) $x+1,-y+1, z+\frac{1}{2} ; \quad$ (v) $x+1, y, z$; (vi) $x,-y+1, z+\frac{1}{2}$; (vii) $x-1, y, z$; (viii) $x, y+1, z$

ecule $[\mathrm{O} \cdots \mathrm{O}=2.857$ (1) $\mathrm{A}]$. The water molecules facilitate the formation of bilayers that stack in an $A B A B$ manner sustained by $\pi-\pi$ interactions. The distances between these layers of molecules can be calculated from the distances between the centroids of the aromatic rings of the two molecules and range from 3.3742 (14) to 4.3402 (14) $\AA$. The ratio between classical hydrogen-bond donors and acceptors is $4: 4$ (four donors and one acceptor on the gallic acid molecule and three acceptors on the caffeine molecule).

The different balance of gallic acid and caffeine in GAL3CAF. $6 \mathrm{H}_{2} \mathbf{O}$ affects the donor/acceptor ratio significantly. There are still only four classical hydrogen-bond

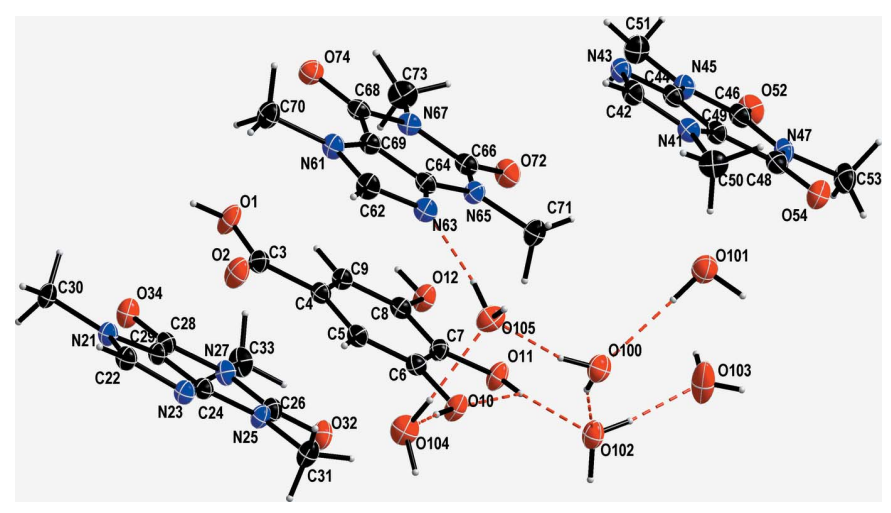

Figure 1

Molecular structure of the GAL3CAF. $6 \mathrm{H}_{2} \mathbf{O}$ showing the labelling scheme and displacement ellipsoids drawn at $50 \%$ probability level. 


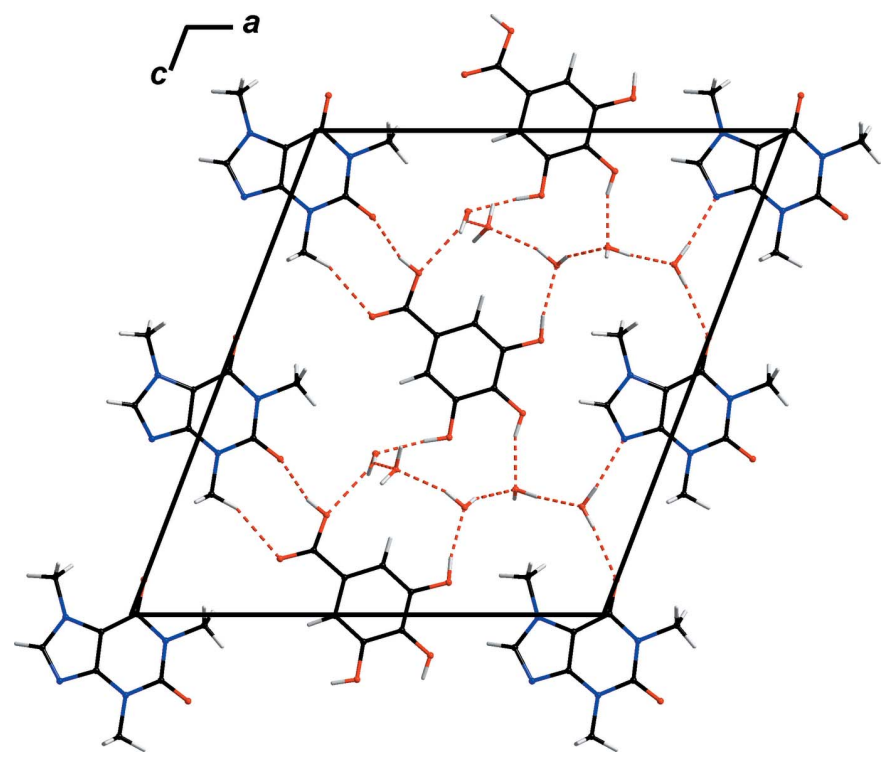

Figure 2

Crystal packing of GAL3CAF $\cdot \mathbf{6 H}_{2} \mathbf{O}$ viewed along $b$. Hydrogen-bonding interactions (Table 1) are shown as red dashed lines.

donors deriving from the hydroxyl groups on gallic acid, but ten hydrogen-bond acceptors (three on each caffeine and one on gallic acid). This discrepency is equilibrated by inclusion of additional solvent water molecules into the crystal structure. These act as structural glue enabling crystallization in different stoichiometries and thus, compensating for the above imbalance. The water molecules provide the additional hydrogen bonds required to form a crystalline solid. Thus, the majority of hydrogen bonds forming the intermolecular network between gallic acid and caffeine are formed by crystal water (Table 1) with $d(D \cdots A)$ ranging from $2.643(2)$ to 3.011 (2) A. Direct classical hydrogen bonding between noncarbon atoms can only be observed between the carboxylic oxygen of gallic acid (O1) and the carbonyl oxygen of caffeine (O32) with $d(D \cdots A)=2.672(2) \AA$ (Fig. 2). Additionally, there is a significant number of weak $\mathrm{C}-\mathrm{H} \cdots \mathrm{O}$ interactions present between gallic acid molecules and caffeine molecules

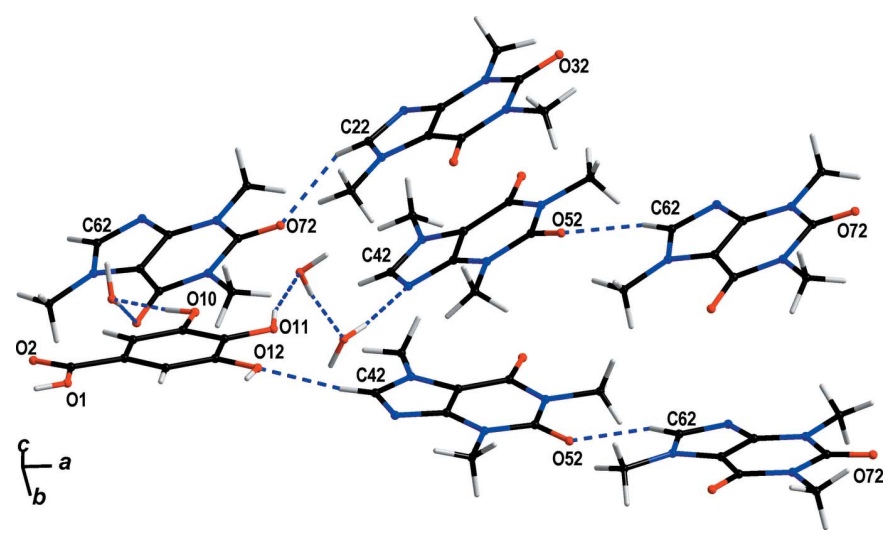

Figure 3

Crystal packing of GAL3CAF $6 \mathbf{H}_{2} \mathbf{O}$. Hydrogen-bonding interactions (Table 1) are shown as blue dashed lines.

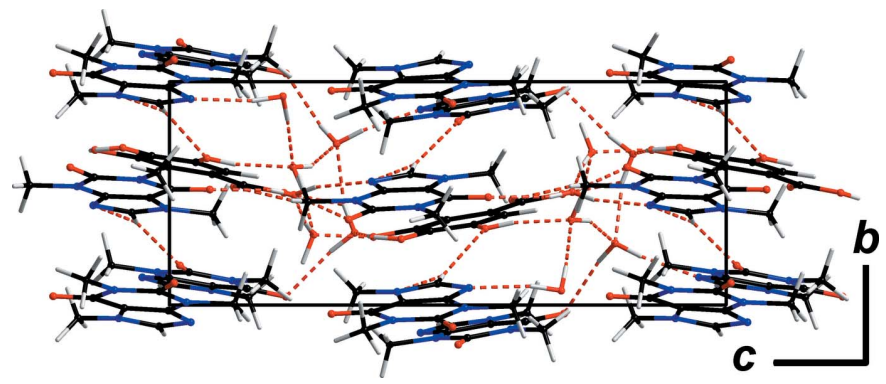

Figure 4

Crystal packing of GAL3CAF. $6 \mathrm{H}_{\mathbf{2}} \mathbf{O}$ viewed along $a$. Hydrogen-bonding interactions are shown as red dashed lines.

and between caffeine molecules themselves (Table 1). One of these interactions is between carboxylic acid and a carbonyl oxygen via hydrogen bonding that is almost parallel to an interaction between the carbonyl oxygen $(\mathrm{O} 2)$ of the carboxylic group in gallic acid with the adjacent proton of a caffeine methyl substituent (C31) $d(D \cdots A) \quad 3.326$ (3) $\AA$. Another $\mathrm{C}-\mathrm{H}$... O interaction is notable as it forms a linear chain connecting all caffeine molecules to each other (Fig. 3). These are formed between the only protonated carbon atom in the purine backbone $(\mathrm{C} 22-\mathrm{H} 22, \mathrm{C} 42-\mathrm{H} 42, \mathrm{C} 62-\mathrm{H} 62)$ and the carbonyl oxygen of the next caffeine molecule (O32, O52, O72) with donor-acceptor distances ranging from 3.119 (3) to 3.227 (3) $\AA$. O12 links gallic acid molecules to these chains via additional weak $\mathrm{C}-\mathrm{H}$. . O interactions to C30 $[d(D \cdots A) 3.247(3) \AA]$ and C42 $[d(D \cdots A)=3.254(3) \AA]$. A comparable interaction between the protonated carbon of the purine ring does not exist in the GALCAF.0.5H structure.

The crystal structure of GAL3CAF. $6 \mathrm{H}_{2} \mathrm{O}$ can be described as having two types of molecular layers connected via hydrogen-bonding interactions with solvent water molecules. Layers consisting solely of caffeine molecules are stacked alternately with layers composed of caffeine and gallic acid molecules (Fig. 4). The distances between the centroids of the aromatic rings are within the significance range at 3.231 (13) and 4.5028 (13) $\AA$. Thus $\pi$-stacking of the aromatic rings is both stronger and weaker in places.

The discussed crystal structure provides a good representation of the large impact of weak $\mathrm{C}-\mathrm{H} \cdots \mathrm{O}$ interactions and of how solvent molecules can play a crucial role in the formation of crystal structures. All our attempts at obtaining a solventless co-crystal with the same stoichiometry have failed so far.

\section{Synthesis and crystallization}

The crystals were obtained as a by-product in a reaction aiming for the synthesis of a lanthanide salt. Gel crystallizations were carried out in order to slow the crystallization process down. This technique involves a piece of glassware that allows two solutions to diffuse through a (tetramethylorthosilicate) gel medium. The two sets of reagents then react when they eventually diffuse through the gel. Tetramethyl- 
Table 2

Experimental details.

\begin{tabular}{|c|c|}
\hline \multicolumn{2}{|l|}{ Crystal data } \\
\hline Chemical formula & $\mathrm{C}_{7} \mathrm{H}_{6} \mathrm{O}_{5} \cdot 3 \mathrm{C}_{8} \mathrm{H}_{10} \mathrm{~N}_{4} \mathrm{O}_{2} \cdot 6 \mathrm{H}_{2} \mathrm{O}$ \\
\hline$M_{\mathrm{r}}$ & 860.81 \\
\hline Crystal system, space group & Monoclinic, $P c$ \\
\hline Temperature $(\mathrm{K})$ & 150 \\
\hline$a, b, c(\AA)$ & $\begin{array}{l}16.5434(3), 6.79456(11) \\
18.1390(4)\end{array}$ \\
\hline$\beta\left({ }^{\circ}\right)$ & $110.865(2)$ \\
\hline$V\left(\AA^{3}\right)$ & $1905.21(7)$ \\
\hline$Z$ & 2 \\
\hline Radiation type & Mo $K \alpha$ \\
\hline$\mu\left(\mathrm{mm}^{-1}\right)$ & 0.12 \\
\hline Crystal size (mm) & $0.66 \times 0.37 \times 0.06$ \\
\hline \multicolumn{2}{|l|}{ Data collection } \\
\hline Diffractometer & $\begin{array}{l}\text { Rigaku Oxford Diffraction } \\
\text { Xcalibur, Atlas, Gemini ultra }\end{array}$ \\
\hline Absorption correction & $\begin{array}{l}\text { Analytical (CrysAlis PRO; Rigaku } \\
\text { OD, 2015) }\end{array}$ \\
\hline$T_{\min }, T_{\max }$ & $0.946,0.994$ \\
\hline $\begin{array}{l}\text { No. of measured, independent and } \\
\text { observed }[I>2 \sigma(I)] \text { reflections }\end{array}$ & $38532,9519,8931$ \\
\hline$R_{\text {int }}$ & 0.029 \\
\hline$(\sin \theta / \lambda)_{\max }\left(\AA^{-1}\right)$ & 0.695 \\
\hline \multicolumn{2}{|l|}{ Refinement } \\
\hline$R\left[F^{2}>2 \sigma\left(F^{2}\right)\right], w R\left(F^{2}\right), S$ & $0.033,0.086,1.04$ \\
\hline No. of reflections & 9519 \\
\hline No. of parameters & 645 \\
\hline No. of restraints & 2 \\
\hline $\mathrm{H}$-atom treatment & $\begin{array}{l}\text { H atoms treated by a mixture of } \\
\text { independent and constrained } \\
\text { refinement }\end{array}$ \\
\hline$\Delta \rho_{\max }, \Delta \rho_{\min }\left(\mathrm{e} \AA^{-3}\right)$ & $0.24,-0.23$ \\
\hline Absolute structure & $\begin{array}{l}\text { Flack } x \text { determined using } 4000 \\
\text { quotients }\left[\left(I^{+}\right)-\left(I^{-}\right)\right] /\left[\left(I^{+}\right)+\left(I^{-}\right)\right] \\
\quad \text { (Parsons } \text { et al., 2013) }\end{array}$ \\
\hline Absolute structure parameter & $-0.1(2)$ \\
\hline
\end{tabular}

Computer programs: CrysAlis PRO (Rigaku OD, 2015), SHELXT (Sheldrick, 2015a), SHELXL (Sheldrick, 2015b), DIAMOND (Brandenburg, 1999) and OLEX2 (Dolomanov et al., 2009).

orthosilicate gel (10\%) was prepared freshly from $7 \mathrm{~mL}$ in $63 \mathrm{~mL}$ distilled water using $\mathrm{Na}_{2} \mathrm{CO}_{3}$ to make the gel approximately $\mathrm{pH} 8$, and left to set overnight using U-tubes. Solutions were put into the two reservoirs: one contained caffeine ( $1 \mathrm{mmol}, 0.2 \mathrm{~g}$ ), while a solution containing an excess gallic acid and lanthanide was in the other.

\section{Refinement}

Crystal data, data collection and structure refinement details are summarized in Table 2. Methyl $\mathrm{H}$ atoms were refined as riding $\left(\mathrm{C}-\mathrm{H}=0.98 \AA\right.$ with $U_{\text {iso }}(\mathrm{H})=1.5 U_{\text {eq }}(\mathrm{C})$.

\section{Funding information}

Funding for this research was provided by: Seventh Framework Programme, FP7 People: Marie-Curie Actions (grant No. 256547 to U. Baisch).

\section{References}

Brandenburg, K. (1999). DIAMOND. Crystal Impact GbR, Bonn, Germany.

Clarke, H. D., Arora, K. K., Bass, H., Kavuru, P., Ong, T. T., Pujari, T., Wojtas, L. \& Zaworotko, M. J. (2010). Cryst. Growth Des. 10, 2152 2167.

Clarke, H. D., Arora, K. K., Wojtas, Ł. \& Zaworotko, M. J. (2011). Cryst. Growth Des. 11, 964-966.

Dolomanov, O. V., Bourhis, L. J., Gildea, R. J., Howard, J. A. K. \& Puschmann, H. (2009). J. Appl. Cryst. 42, 339-341.

Groom, C. R., Bruno, I. J., Lightfoot, M. P. \& Ward, S. C. (2016). Acta Cryst. B72, 171-179.

Nayeem, N., Asdaq, S. M. B., Salem, H. \& AHEl-Alfqy, S. (2016). J. App. Pharm. 8, 213.

Parsons, S., Flack, H. D. \& Wagner, T. (2013). Acta Cryst. B69, 249 259.

Rigaku OD (2015). CrysAlis PRO. Rigaku Oxford Diffraction, Yarnton, England.

Sheldrick, G. M. (2015a). Acta Cryst. A71, 3-8.

Sheldrick, G. M. (2015b). Acta Cryst. C71, 3-8.

Steiner, T. (2002). Angew. Chem. Int. Ed. 41, 48-76.

Zhao, X., Zhang, W., Kong, S. J., Zheng, X., Zheng, J. \& Shi, R. (2007). J. Liq. Chromatogr. Related Technol. 30, 235-244. 


\section{supporting information}

Acta Cryst. (2018). E74, 559-562 [https://doi.org/10.1107/S2056989018004528]

\section{Crystal water as the molecular glue for obtaining different co-crystal ratios: the case of gallic acid tris-caffeine hexahydrate}

\section{Vella-Zarb and U. Baisch}

Computing details

Data collection: CrysAlis PRO (Rigaku OD, 2015); cell refinement: CrysAlis PRO (Rigaku OD, 2015); data reduction: CrysAlis PRO (Rigaku OD, 2015); program(s) used to solve structure: SHELXT (Sheldrick, 2015a); program(s) used to refine structure: SHELXL (Sheldrick, 2015b); molecular graphics: DIAMOND (Brandenburg, 1999); software used to prepare material for publication: OLEX2 (Dolomanov et al., 2009).

Gallic Acid Tris-Caffeine hexahydrate

Crystal data

$\mathrm{C}_{7} \mathrm{H}_{6} \mathrm{O}_{5} \cdot 3 \mathrm{C}_{8} \mathrm{H}_{10} \mathrm{~N}_{4} \mathrm{O}_{2} \cdot 6 \mathrm{H}_{2} \mathrm{O}$

$M_{r}=860.81$

Monoclinic, $P c$

$a=16.5434$ (3) $\AA$

$b=6.79456(11) \AA$

$c=18.1390$ (4) $\AA$

$\beta=110.865(2)^{\circ}$

$V=1905.21(7) \AA^{3}$

$Z=2$

\section{Data collection}

Rigaku Oxford Diffraction Xcalibur, Atlas, Gemini ultra diffractometer

Detector resolution: 10.3968 pixels $\mathrm{mm}^{-1}$ $\omega$ scans

Absorption correction: analytical

(CrysAlisPro; Rigaku OD, 2015)

$T_{\min }=0.946, T_{\max }=0.994$

Refinement

Refinement on $F^{2}$

Least-squares matrix: full

$R\left[F^{2}>2 \sigma\left(F^{2}\right)\right]=0.033$

$w R\left(F^{2}\right)=0.086$

$S=1.04$

9519 reflections

645 parameters

2 restraints

Primary atom site location: dual

Hydrogen site location: mixed
$F(000)=908$

$D_{\mathrm{x}}=1.501 \mathrm{Mg} \mathrm{m}^{-3}$

Mo $K \alpha$ radiation, $\lambda=0.71073 \AA$

Cell parameters from 27647 reflections

$\theta=3.0-29.6^{\circ}$

$\mu=0.12 \mathrm{~mm}^{-1}$

$T=150 \mathrm{~K}$

Plate, clear colourless

$0.66 \times 0.37 \times 0.06 \mathrm{~mm}$

38532 measured reflections

9519 independent reflections

8931 reflections with $I>2 \sigma(I)$

$R_{\text {int }}=0.029$

$\theta_{\max }=29.6^{\circ}, \theta_{\min }=3.0^{\circ}$

$h=-21 \rightarrow 22$

$k=-9 \rightarrow 9$

$l=-25 \rightarrow 24$

$\mathrm{H}$ atoms treated by a mixture of independent and constrained refinement

$w=1 /\left[\sigma^{2}\left(F_{\mathrm{o}}^{2}\right)+(0.051 P)^{2}+0.2608 P\right]$

where $P=\left(F_{\mathrm{o}}^{2}+2 F_{\mathrm{c}}{ }^{2}\right) / 3$

$(\Delta / \sigma)_{\max }<0.001$

$\Delta \rho_{\max }=0.24 \mathrm{e} \AA^{-3}$

$\Delta \rho_{\min }=-0.23$ e $\AA^{-3}$

Absolute structure: Flack $x$ determined using 4000 quotients $\left[\left(I^{+}\right)-(I)\right] /\left[\left(I^{+}\right)+\left(I^{-}\right)\right]$(Parsons et al., 2013)

Absolute structure parameter: -0.1 (2) 


\section{Special details}

Geometry. All esds (except the esd in the dihedral angle between two 1.s. planes) are estimated using the full covariance matrix. The cell esds are taken into account individually in the estimation of esds in distances, angles and torsion angles; correlations between esds in cell parameters are only used when they are defined by crystal symmetry. An approximate (isotropic) treatment of cell esds is used for estimating esds involving l.s. planes.

Fractional atomic coordinates and isotropic or equivalent isotropic displacement parameters $\left(\hat{A}^{2}\right)$

\begin{tabular}{|c|c|c|c|c|}
\hline & $x$ & $y$ & $z$ & $U_{\text {iso }} * / U_{\text {eq }}$ \\
\hline $\mathrm{O} 12$ & $0.64601(10)$ & $0.3426(2)$ & $0.43298(9)$ & $0.0237(3)$ \\
\hline O74 & $0.39816(11)$ & $0.9622(2)$ & $0.29064(9)$ & $0.0249(3)$ \\
\hline $\mathrm{O} 10$ & $0.53021(10)$ & $0.3054(3)$ & $0.63346(9)$ & $0.0250(3)$ \\
\hline $\mathrm{O} 72$ & $0.66076(10)$ & $0.8227(3)$ & $0.48111(10)$ & $0.0279(3)$ \\
\hline O102 & $0.71299(11)$ & $0.3149(3)$ & $0.74098(9)$ & $0.0259(3)$ \\
\hline O11 & $0.66635(9)$ & $0.2955(2)$ & $0.58370(9)$ & $0.0241(3)$ \\
\hline $\mathrm{O} 32$ & $0.19013(10)$ & $0.3831(2)$ & $0.67800(9)$ & $0.0257(3)$ \\
\hline $\mathrm{O} 34$ & $-0.00090(10)$ & $0.4828(2)$ & $0.42738(9)$ & $0.0259(3)$ \\
\hline O54 & $1.06258(11)$ & $0.9604(3)$ & $0.70872(10)$ & $0.0289(3)$ \\
\hline $\mathrm{O} 1$ & $0.33315(11)$ & $0.4973(3)$ & $0.29402(10)$ & $0.0292(4)$ \\
\hline O100 & $0.61941(12)$ & $0.6181(3)$ & $0.77785(10)$ & $0.0266(3)$ \\
\hline O105 & $0.44257(11)$ & $0.7234(3)$ & $0.69929(10)$ & $0.0288(3)$ \\
\hline $\mathrm{O} 52$ & $1.14078(10)$ & $0.9016(3)$ & $0.49147(10)$ & $0.0300(4)$ \\
\hline $\mathrm{O} 2$ & $0.27018(10)$ & $0.4707(3)$ & $0.38411(10)$ & $0.0300(4)$ \\
\hline O104 & $0.38756(11)$ & $0.3238(3)$ & $0.66710(11)$ & $0.0312(4)$ \\
\hline O101 & $0.74791(12)$ & $0.9351(3)$ & $0.79622(11)$ & $0.0325(4)$ \\
\hline N27 & $0.09392(11)$ & $0.4384(3)$ & $0.55358(11)$ & $0.0200(3)$ \\
\hline N41 & $0.88046(12)$ & $1.0593(3)$ & $0.59097(11)$ & $0.0210(3)$ \\
\hline N23 & $-0.09763(11)$ & $0.5534(3)$ & $0.63666(10)$ & $0.0203(3)$ \\
\hline N67 & $0.52922(11)$ & $0.8905(3)$ & $0.38714(11)$ & $0.0201(3)$ \\
\hline N21 & $-0.13658(11)$ & $0.5677(3)$ & $0.50514(10)$ & $0.0187(3)$ \\
\hline N61 & $0.32462(12)$ & $0.9330(3)$ & $0.42452(11)$ & $0.0216(4)$ \\
\hline N63 & $0.41353(12)$ & $0.8759(3)$ & $0.54870(11)$ & $0.0221(4)$ \\
\hline O103 & $0.86742(14)$ & $0.4935(4)$ & $0.77642(12)$ & $0.0486(6)$ \\
\hline N45 & $1.00201(12)$ & $0.9877(3)$ & $0.46922(11)$ & $0.0213(4)$ \\
\hline N47 & $1.10076(11)$ & $0.9323(3)$ & $0.59887(11)$ & $0.0221(4)$ \\
\hline N43 & $0.85646(11)$ & $1.0770(3)$ & $0.46163(11)$ & $0.0223(4)$ \\
\hline $\mathrm{N} 25$ & $0.05332(11)$ & $0.4685(3)$ & $0.66583(10)$ & $0.0200(3)$ \\
\hline N65 & $0.54639(11)$ & $0.8526(3)$ & $0.52143(10)$ & $0.0193(3)$ \\
\hline C9 & $0.49273(13)$ & $0.4032(3)$ & $0.39853(12)$ & $0.0182(4)$ \\
\hline $\mathrm{C} 28$ & $0.01160(13)$ & $0.4814(3)$ & $0.49805(12)$ & $0.0185(4)$ \\
\hline C68 & $0.43992(14)$ & $0.9264(3)$ & $0.36060(12)$ & $0.0193(4)$ \\
\hline C64 & $0.45928(13)$ & $0.8801(3)$ & $0.49996(12)$ & $0.0188(4)$ \\
\hline $\mathrm{C} 5$ & $0.43204(13)$ & $0.3818(3)$ & $0.50144(12)$ & $0.0185(4)$ \\
\hline $\mathrm{C} 22$ & $-0.16156(14)$ & $0.5854(3)$ & $0.56754(12)$ & $0.0204(4)$ \\
\hline $\mathrm{C} 4$ & 0.42193 (13) & $0.4139(3)$ & $0.42310(12)$ & $0.0180(4)$ \\
\hline $\mathrm{C} 26$ & $0.11628(13)$ & $0.4278(3)$ & $0.63532(12)$ & $0.0198(4)$ \\
\hline C3 & $0.33445(14)$ & $0.4627(3)$ & $0.36677(12)$ & $0.0202(4)$ \\
\hline $\mathrm{C} 42$ & $0.82336(14)$ & $1.0955(3)$ & $0.51858(13)$ & $0.0227(4)$ \\
\hline
\end{tabular}




\begin{tabular}{|c|c|c|c|c|}
\hline C62 & $0.33233(14)$ & $0.9082(3)$ & $0.49978(13)$ & $0.0241(4)$ \\
\hline C6 & $0.51354(13)$ & $0.3398(3)$ & $0.55560(12)$ & 0.0189 (4) \\
\hline $\mathrm{C} 29$ & $-0.04957(13)$ & $0.5192(3)$ & $0.53528(12)$ & $0.0181(4)$ \\
\hline $\mathrm{C} 24$ & $-0.02878(13)$ & $0.5128(3)$ & $0.61509(12)$ & $0.0182(4)$ \\
\hline $\mathrm{C} 8$ & $0.57394(13)$ & $0.3606(3)$ & $0.45261(12)$ & $0.0184(4)$ \\
\hline C69 & $0.40732(13)$ & $0.9150(3)$ & $0.42277(12)$ & $0.0185(4)$ \\
\hline $\mathrm{C} 7$ & $0.58546(13)$ & $0.3319(3)$ & $0.53187(12)$ & $0.0183(4)$ \\
\hline C49 & $0.95774(13)$ & $1.0134(3)$ & $0.58130(13)$ & $0.0190(4)$ \\
\hline $\mathrm{C} 48$ & $1.04131(14)$ & $0.9686(3)$ & 0.63709 (13) & $0.0217(4)$ \\
\hline $\mathrm{C} 46$ & $1.08429(14)$ & $0.9386(3)$ & $0.51791(13)$ & $0.0219(4)$ \\
\hline C30 & $-0.19092(13)$ & 0.5917 (3) & $0.42252(12)$ & $0.0208(4)$ \\
\hline $\mathrm{H} 30 \mathrm{~A}$ & -0.195314 & 0.465826 & 0.394977 & $0.031 *$ \\
\hline H30B & -0.165253 & 0.690518 & 0.398138 & $0.031 *$ \\
\hline $\mathrm{H} 30 \mathrm{C}$ & -0.248743 & 0.634908 & 0.418844 & $0.031 *$ \\
\hline $\mathrm{C} 44$ & 0.94005 (13) & $1.0249(3)$ & $0.50165(12)$ & $0.0197(4)$ \\
\hline C66 & $0.58361(14)$ & $0.8529(3)$ & 0.46485 (13) & $0.0206(4)$ \\
\hline C71 & $0.60075(14)$ & $0.8227(3)$ & $0.60429(13)$ & $0.0258(4)$ \\
\hline H71A & 0.578830 & 0.903248 & 0.637924 & $0.039 *$ \\
\hline H71B & 0.660364 & 0.861399 & 0.612352 & $0.039 *$ \\
\hline $\mathrm{H} 71 \mathrm{C}$ & 0.599411 & 0.683589 & 0.618036 & $0.039^{*}$ \\
\hline C31 & $0.07288(15)$ & $0.4634(4)$ & $0.75120(13)$ & $0.0263(4)$ \\
\hline $\mathrm{H} 31 \mathrm{~A}$ & 0.049780 & 0.341920 & 0.765162 & $0.039 *$ \\
\hline H31B & 0.046261 & 0.577200 & 0.766781 & $0.039^{*}$ \\
\hline $\mathrm{H} 31 \mathrm{C}$ & 0.135692 & 0.467660 & 0.778760 & $0.039^{*}$ \\
\hline C51 & $0.98219(16)$ & $0.9950(4)$ & $0.38378(14)$ & $0.0280(5)$ \\
\hline $\mathrm{H} 51 \mathrm{~A}$ & 1.028745 & 1.063988 & 0.372886 & $0.042 *$ \\
\hline H51B & 0.927512 & 1.065112 & 0.358458 & $0.042 *$ \\
\hline $\mathrm{H} 51 \mathrm{C}$ & 0.977081 & 0.860691 & 0.362901 & $0.042 *$ \\
\hline $\mathrm{C} 50$ & $0.86261(17)$ & $1.0583(4)$ & $0.66409(15)$ & $0.0284(5)$ \\
\hline $\mathrm{C} 33$ & $0.16380(14)$ & $0.4060(4)$ & $0.52176(14)$ & $0.0259(5)$ \\
\hline H33A & 0.148733 & 0.294353 & 0.485177 & $0.039 *$ \\
\hline H33B & 0.218050 & 0.377880 & 0.565271 & $0.039^{*}$ \\
\hline $\mathrm{H} 33 \mathrm{C}$ & 0.170850 & 0.524415 & 0.493788 & $0.039^{*}$ \\
\hline C53 & 1.19054 (14) & $0.8887(4)$ & $0.64940(15)$ & $0.0301(5)$ \\
\hline $\mathrm{H} 53 \mathrm{~A}$ & 1.225454 & 1.008608 & 0.656930 & $0.045^{*}$ \\
\hline H53B & 1.214230 & 0.787698 & 0.624216 & $0.045^{*}$ \\
\hline H53C & 1.191737 & 0.840579 & 0.700719 & $0.045^{*}$ \\
\hline C73 & $0.57111(16)$ & $0.8896(4)$ & $0.32820(15)$ & $0.0292(5)$ \\
\hline $\mathrm{H} 73 \mathrm{~A}$ & 0.573261 & 0.754593 & 0.309962 & $0.044 *$ \\
\hline H73B & 0.630055 & 0.941262 & 0.351905 & $0.044^{*}$ \\
\hline $\mathrm{H} 73 \mathrm{C}$ & 0.538002 & 0.972313 & 0.283348 & $0.044 *$ \\
\hline H5 & 0.3845 (19) & $0.391(4)$ & $0.5203(16)$ & $0.022(6)^{*}$ \\
\hline H9 & $0.4873(17)$ & $0.424(4)$ & $0.3426(16)$ & $0.017(6)^{*}$ \\
\hline $\mathrm{H} 22$ & $-0.220(2)$ & $0.629(4)$ & $0.5596(17)$ & $0.030(7)^{*}$ \\
\hline $\mathrm{H} 1$ & $0.282(2)$ & $0.521(5)$ & $0.2629(19)$ & $0.035(8)^{*}$ \\
\hline H12 & $0.631(2)$ & $0.369(4)$ & $0.3832(19)$ & $0.031(7)^{*}$ \\
\hline H62 & $0.284(2)$ & $0.912(5)$ & $0.5170(18)$ & $0.035(8)^{*}$ \\
\hline H10 & $0.482(3)$ & $0.308(6)$ & $0.641(2)$ & $0.061(11)^{*}$ \\
\hline
\end{tabular}




$\begin{array}{lllll}\text { C70 } & 0.24418(15) & 0.9659(4) & 0.35795(14) & 0.0265(4) \\ \text { H70A } & 0.194877 & 0.947555 & 0.375199 & 0.040^{*} \\ \text { H70B } & 0.240186 & 0.871975 & 0.315813 & 0.040^{*} \\ \text { H70C } & 0.243510 & 1.100411 & 0.338199 & 0.040^{*} \\ \text { H42 } & 0.767(2) & 1.131(4) & 0.5092(18) & 0.033(7)^{*} \\ \text { H11 } & 0.669(2) & 0.304(5) & 0.630(2) & 0.041(9)^{*} \\ \text { H10G } & 0.392(2) & 0.449(5) & 0.6864(19) & 0.036(8)^{*} \\ \text { H10E } & 0.425(3) & 0.802(6) & 0.728(3) & 0.062(11)^{*} \\ \text { H10F } & 0.436(2) & 0.781(6) & 0.654(2) & 0.053(10)^{*} \\ \text { H10A } & 0.718(2) & 0.192(6) & 0.760(2) & 0.056(10)^{*} \\ \text { H10I } & 0.781(2) & 0.915(5) & 0.850(2) & 0.039(8)^{*} \\ \text { H10C } & 0.568(3) & 0.622(6) & 0.746(2) & 0.055(11)^{*} \\ \text { H10D } & 0.634(2) & 0.518(5) & 0.7604(19) & 0.034(8)^{*} \\ \text { H10B } & 0.763(2) & 0.367(5) & 0.7558(19) & 0.033(8)^{*} \\ \text { H10K } & 0.905(3) & 0.515(7) & 0.816(3) & 0.076(14)^{*} \\ \text { H10L } & 0.881(3) & 0.528(6) & 0.738(3) & 0.072(13)^{*} \\ \text { H10H } & 0.389(3) & 0.243(7) & 0.709(3) & 0.077(13)^{*} \\ \text { H10J } & 0.714(3) & 0.832(6) & 0.788(2) & 0.057(11)^{*} \\ \text { H50A } & 0.868(2) & 0.928(5) & 0.683(2) & 0.044(9)^{*} \\ \text { H50B } & 0.803(3) & 1.094(6) & 0.651(2) & 0.063(11)^{*} \\ \text { H50C } & 0.902(3) & 1.156(5) & 0.701(2) & 0.055(10)^{*} \\ \end{array}$

Atomic displacement parameters $\left(\AA^{2}\right)$

\begin{tabular}{|c|c|c|c|c|c|c|}
\hline & $U^{11}$ & $U^{22}$ & $U^{33}$ & $U^{12}$ & $U^{13}$ & $U^{23}$ \\
\hline $\mathrm{O} 12$ & $0.0148(7)$ & $0.0379(8)$ & $0.0204(7)$ & $0.0037(6)$ & $0.0089(6)$ & $0.0030(6)$ \\
\hline $\mathrm{O} 74$ & $0.0245(8)$ & $0.0298(7)$ & $0.0219(7)$ & $-0.0003(6)$ & $0.0102(6)$ & $0.0024(6)$ \\
\hline $\mathrm{O} 10$ & $0.0172(7)$ & $0.0413(9)$ & $0.0174(7)$ & $0.0025(6)$ & $0.0073(6)$ & $0.0042(6)$ \\
\hline $\mathrm{O} 72$ & $0.0165(7)$ & $0.0332(8)$ & $0.0358(9)$ & $0.0021(6)$ & $0.0117(6)$ & $-0.0004(7)$ \\
\hline O102 & $0.0174(8)$ & $0.0367(9)$ & $0.0231(7)$ & $-0.0010(7)$ & $0.0065(6)$ & $-0.0007(7)$ \\
\hline $\mathrm{O} 11$ & $0.0138(7)$ & $0.0391(9)$ & $0.0191(7)$ & $0.0056(6)$ & $0.0055(6)$ & $0.0018(6)$ \\
\hline $\mathrm{O} 32$ & $0.0144(7)$ & $0.0329(8)$ & $0.0264(8)$ & $0.0030(6)$ & $0.0030(6)$ & $-0.0036(6)$ \\
\hline $\mathrm{O} 34$ & $0.0196(7)$ & $0.0384(9)$ & $0.0206(7)$ & $0.0012(6)$ & $0.0081(6)$ & $-0.0031(6)$ \\
\hline O54 & $0.0235(8)$ & $0.0373(8)$ & $0.0233(8)$ & $0.0024(7)$ & $0.0049(7)$ & $-0.0005(7)$ \\
\hline $\mathrm{O} 1$ & $0.0149(7)$ & $0.0485(10)$ & $0.0214(7)$ & $0.0049(7)$ & $0.0031(6)$ & $0.0077(7)$ \\
\hline O100 & $0.0244(9)$ & $0.0339(9)$ & $0.0214(8)$ & $0.0019(7)$ & $0.0081(7)$ & $-0.0022(7)$ \\
\hline O105 & $0.0314(9)$ & $0.0353(9)$ & $0.0236(8)$ & $0.0057(7)$ & $0.0145(7)$ & $0.0029(7)$ \\
\hline O52 & $0.0197(8)$ & $0.0368(9)$ & $0.0378(9)$ & $0.0017(6)$ & $0.0154(7)$ & $-0.0023(7)$ \\
\hline $\mathrm{O} 2$ & $0.0150(7)$ & $0.0467(10)$ & $0.0282(8)$ & $0.0038(7)$ & $0.0075(7)$ & $0.0045(7)$ \\
\hline O104 & $0.0268(8)$ & $0.0426(10)$ & $0.0290(8)$ & $0.0020(7)$ & $0.0159(7)$ & $0.0003(8)$ \\
\hline O101 & $0.0279(9)$ & $0.0394(10)$ & $0.0288(9)$ & $-0.0025(8)$ & $0.0085(7)$ & $0.0033(7)$ \\
\hline N27 & $0.0135(8)$ & $0.0242(8)$ & $0.0233(9)$ & $0.0001(6)$ & $0.0078(7)$ & $-0.0025(6)$ \\
\hline N41 & $0.0172(8)$ & $0.0218(8)$ & $0.0262(9)$ & $0.0010(7)$ & $0.0104(7)$ & $-0.0017(7)$ \\
\hline $\mathrm{N} 23$ & $0.0164(8)$ & $0.0250(8)$ & $0.0203(8)$ & $0.0000(7)$ & $0.0074(7)$ & $-0.0001(7)$ \\
\hline N67 & $0.0185(9)$ & $0.0222(8)$ & $0.0236(8)$ & $0.0005(7)$ & $0.0122(7)$ & $0.0006(7)$ \\
\hline N21 & $0.0128(8)$ & $0.0232(8)$ & $0.0200(8)$ & $0.0001(6)$ & $0.0058(7)$ & $-0.0005(6)$ \\
\hline N61 & $0.0144(8)$ & $0.0278(9)$ & $0.0245(9)$ & $-0.0001(7)$ & $0.0094(7)$ & $0.0000(7)$ \\
\hline N63 & $0.0190(9)$ & $0.0281(9)$ & $0.0224(8)$ & $-0.0001(7)$ & $0.0111(7)$ & $0.0008(7)$ \\
\hline
\end{tabular}




\begin{tabular}{|c|c|c|c|c|c|c|}
\hline O103 & $0.0296(10)$ & $0.0955(18)$ & $0.0216(9)$ & $-0.0251(11)$ & $0.0102(8)$ & $-0.0029(10)$ \\
\hline N45 & $0.0164(8)$ & $0.0253(9)$ & $0.0234(9)$ & $0.0010(7)$ & $0.0086(7)$ & $-0.0011(7)$ \\
\hline N47 & $0.0121(8)$ & $0.0251(8)$ & $0.0270(9)$ & $0.0005(7)$ & $0.0046(7)$ & $-0.0026(7)$ \\
\hline N43 & 0.0155 (9) & $0.0225(8)$ & $0.0268(9)$ & $0.0010(6)$ & $0.0047(7)$ & $-0.0012(7)$ \\
\hline N25 & $0.0146(8)$ & $0.0250(8)$ & $0.0187(8)$ & $0.0013(6)$ & 0.0038 (7) & $0.0010(6)$ \\
\hline N65 & $0.0147(8)$ & $0.0231(8)$ & $0.0210(8)$ & $0.0011(6)$ & $0.0073(7)$ & $0.0015(7)$ \\
\hline C9 & $0.0166(9)$ & $0.0211(9)$ & 0.0177 (9) & $-0.0003(7)$ & $0.0073(8)$ & $0.0000(7)$ \\
\hline $\mathrm{C} 28$ & $0.0156(9)$ & $0.0194(9)$ & 0.0208 (9) & $-0.0013(7)$ & $0.0066(8)$ & $-0.0014(7)$ \\
\hline C68 & $0.0194(10)$ & $0.0170(9)$ & $0.0242(10)$ & $-0.0007(7)$ & $0.0110(8)$ & $-0.0004(7)$ \\
\hline C64 & $0.0168(9)$ & $0.0189(9)$ & $0.0223(10)$ & $-0.0015(7)$ & $0.0090(8)$ & $-0.0010(7)$ \\
\hline $\mathrm{C} 5$ & 0.0139 (9) & 0.0215 (9) & $0.0217(10)$ & $-0.0007(7)$ & $0.0084(8)$ & $-0.0001(7)$ \\
\hline $\mathrm{C} 22$ & $0.0156(10)$ & $0.0245(10)$ & $0.0221(10)$ & $-0.0005(8)$ & $0.0078(8)$ & $-0.0002(8)$ \\
\hline $\mathrm{C} 4$ & $0.0151(9)$ & $0.0179(9)$ & $0.0211(9)$ & $-0.0003(7)$ & $0.0066(8)$ & $-0.0006(7)$ \\
\hline $\mathrm{C} 26$ & $0.0152(9)$ & $0.0204(9)$ & $0.0227(10)$ & $-0.0003(7)$ & $0.0053(8)$ & $-0.0022(8)$ \\
\hline $\mathrm{C} 3$ & $0.0161(9)$ & $0.0222(9)$ & $0.0214(10)$ & $0.0000(7)$ & $0.0056(8)$ & $0.0008(8)$ \\
\hline $\mathrm{C} 42$ & $0.0134(10)$ & $0.0236(10)$ & $0.0307(11)$ & $0.0011(7)$ & $0.0076(8)$ & $-0.0013(8)$ \\
\hline C62 & $0.0176(10)$ & $0.0329(11)$ & $0.0252(10)$ & $0.0001(8)$ & $0.0118(8)$ & $0.0008(8)$ \\
\hline C6 & $0.0187(9)$ & $0.0199(9)$ & $0.0190(9)$ & $-0.0013(7)$ & $0.0078(8)$ & $-0.0009(7)$ \\
\hline $\mathrm{C} 29$ & 0.0128 (9) & $0.0200(9)$ & $0.0206(9)$ & $-0.0006(7)$ & $0.0050(7)$ & $0.0001(7)$ \\
\hline $\mathrm{C} 24$ & $0.0146(9)$ & $0.0180(9)$ & $0.0218(10)$ & $0.0000(7)$ & $0.0063(8)$ & $0.0003(7)$ \\
\hline $\mathrm{C} 8$ & 0.0158 (9) & $0.0196(9)$ & $0.0222(10)$ & $-0.0003(7)$ & $0.0097(8)$ & $-0.0005(7)$ \\
\hline C69 & $0.0151(9)$ & $0.0193(9)$ & $0.0225(10)$ & $0.0004(7)$ & $0.0086(8)$ & $0.0003(7)$ \\
\hline $\mathrm{C} 7$ & $0.0145(9)$ & $0.0196(9)$ & $0.0193(9)$ & $0.0016(7)$ & $0.0042(7)$ & $-0.0001(7)$ \\
\hline $\mathrm{C} 49$ & $0.0132(9)$ & $0.0206(9)$ & $0.0238(10)$ & $0.0003(7)$ & $0.0072(8)$ & $-0.0015(7)$ \\
\hline $\mathrm{C} 48$ & $0.0178(10)$ & $0.0189(9)$ & $0.0282(11)$ & $0.0002(8)$ & 0.0078 (9) & $-0.0021(8)$ \\
\hline $\mathrm{C} 46$ & $0.0167(10)$ & $0.0204(9)$ & $0.0291(11)$ & $-0.0007(7)$ & $0.0089(8)$ & $-0.0022(8)$ \\
\hline $\mathrm{C} 30$ & $0.0144(9)$ & $0.0281(10)$ & $0.0175(9)$ & $0.0007(7)$ & $0.0028(8)$ & $0.0003(8)$ \\
\hline $\mathrm{C} 44$ & $0.0159(9)$ & $0.0184(9)$ & $0.0253(10)$ & $-0.0011(7)$ & $0.0078(8)$ & $-0.0025(8)$ \\
\hline C66 & $0.0187(9)$ & $0.0181(9)$ & $0.0271(10)$ & $0.0000(7)$ & $0.0107(8)$ & $-0.0003(8)$ \\
\hline C71 & $0.0199(10)$ & $0.0316(11)$ & $0.0235(10)$ & 0.0017 (9) & $0.0048(8)$ & $0.0005(9)$ \\
\hline C31 & $0.0205(10)$ & $0.0381(12)$ & $0.0182(10)$ & $0.0022(9)$ & $0.0044(8)$ & $0.0024(9)$ \\
\hline $\mathrm{C} 51$ & $0.0279(12)$ & $0.0360(12)$ & $0.0226(10)$ & $-0.0008(9)$ & $0.0119(9)$ & $-0.0024(9)$ \\
\hline $\mathrm{C} 50$ & $0.0280(12)$ & $0.0328(12)$ & $0.0312(12)$ & $0.0035(10)$ & $0.0190(10)$ & $-0.0003(10)$ \\
\hline $\mathrm{C} 33$ & $0.0148(10)$ & 0.0335 (11) & $0.0327(12)$ & $0.0018(8)$ & $0.0125(9)$ & -0.0035 (9) \\
\hline $\mathrm{C} 53$ & $0.0146(10)$ & $0.0324(11)$ & $0.0376(13)$ & $0.0031(8)$ & $0.0023(9)$ & $-0.0039(10)$ \\
\hline $\mathrm{C} 73$ & $0.0266(12)$ & $0.0373(12)$ & $0.0315(12)$ & $0.0016(9)$ & $0.0198(10)$ & $0.0014(10)$ \\
\hline C70 & $0.0175(10)$ & $0.0350(11)$ & $0.0255(10)$ & $0.0019(9)$ & $0.0056(8)$ & $0.0018(9)$ \\
\hline
\end{tabular}

Geometric parameters $\left(A,{ }^{\circ}\right)$

\begin{tabular}{llll}
\hline $\mathrm{O} 12-\mathrm{C} 8$ & $1.366(2)$ & $\mathrm{N} 43-\mathrm{C} 44$ & $1.360(3)$ \\
$\mathrm{O} 12-\mathrm{H} 12$ & $0.86(3)$ & $\mathrm{N} 25-\mathrm{C} 26$ & $1.370(3)$ \\
$\mathrm{O} 74-\mathrm{C} 68$ & $1.234(3)$ & $\mathrm{N} 25-\mathrm{C} 24$ & $1.376(3)$ \\
$\mathrm{O} 10-\mathrm{C} 6$ & $1.359(2)$ & $\mathrm{N} 25-\mathrm{C} 31$ & $1.465(3)$ \\
$\mathrm{O} 10-\mathrm{H} 10$ & $0.86(4)$ & $\mathrm{N} 65-\mathrm{C} 64$ & $1.365(3)$ \\
$\mathrm{O} 72-\mathrm{C} 66$ & $1.221(3)$ & $\mathrm{N} 65-\mathrm{C} 66$ & $1.372(3)$ \\
$\mathrm{O} 102-\mathrm{H} 10 \mathrm{~A}$ & $0.89(4)$ & $\mathrm{N} 65-\mathrm{C} 71$ & $1.465(3)$ \\
$\mathrm{O} 102-\mathrm{H} 10 \mathrm{~B}$ & $0.85(4)$ & $\mathrm{C} 9-\mathrm{C} 4$ & $1.396(3)$
\end{tabular}




\begin{tabular}{|c|c|c|c|}
\hline $\mathrm{O} 11-\mathrm{C} 7$ & $1.356(2)$ & $\mathrm{C} 9-\mathrm{C} 8$ & $1.383(3)$ \\
\hline O11-H11 & $0.83(4)$ & $\mathrm{C} 9-\mathrm{H} 9$ & $1.00(3)$ \\
\hline $\mathrm{O} 32-\mathrm{C} 26$ & $1.229(3)$ & $\mathrm{C} 28-\mathrm{C} 29$ & $1.426(3)$ \\
\hline $\mathrm{O} 34-\mathrm{C} 28$ & $1.224(3)$ & C68-C69 & $1.415(3)$ \\
\hline $\mathrm{O} 54-\mathrm{C} 48$ & $1.221(3)$ & C64-C69 & $1.378(3)$ \\
\hline $\mathrm{O} 1-\mathrm{C} 3$ & $1.333(3)$ & $\mathrm{C} 5-\mathrm{C} 4$ & $1.388(3)$ \\
\hline $\mathrm{O} 1-\mathrm{H} 1$ & $0.85(3)$ & $\mathrm{C} 5-\mathrm{C} 6$ & $1.386(3)$ \\
\hline $\mathrm{O} 100-\mathrm{H} 10 \mathrm{C}$ & $0.83(4)$ & $\mathrm{C} 5-\mathrm{H} 5$ & $0.97(3)$ \\
\hline $\mathrm{O} 100-\mathrm{H} 10 \mathrm{D}$ & $0.82(4)$ & $\mathrm{C} 22-\mathrm{H} 22$ & $0.97(3)$ \\
\hline $\mathrm{O} 105-\mathrm{H} 10 \mathrm{E}$ & $0.86(5)$ & $\mathrm{C} 4-\mathrm{C} 3$ & $1.481(3)$ \\
\hline $\mathrm{O} 105-\mathrm{H} 10 \mathrm{~F}$ & $0.88(4)$ & $\mathrm{C} 42-\mathrm{H} 42$ & $0.92(3)$ \\
\hline $\mathrm{O} 52-\mathrm{C} 46$ & $1.219(3)$ & C62-H62 & $0.96(3)$ \\
\hline $\mathrm{O} 2-\mathrm{C} 3$ & $1.213(3)$ & $\mathrm{C} 6-\mathrm{C} 7$ & $1.403(3)$ \\
\hline $\mathrm{O} 104-\mathrm{H} 10 \mathrm{G}$ & $0.91(3)$ & $\mathrm{C} 29-\mathrm{C} 24$ & $1.364(3)$ \\
\hline $\mathrm{O} 104-\mathrm{H} 10 \mathrm{H}$ & $0.93(5)$ & $\mathrm{C} 8-\mathrm{C} 7$ & $1.395(3)$ \\
\hline O101-H10I & $0.94(4)$ & $\mathrm{C} 49-\mathrm{C} 48$ & $1.425(3)$ \\
\hline O101-H10J & $0.88(4)$ & $\mathrm{C} 49-\mathrm{C} 44$ & $1.370(3)$ \\
\hline $\mathrm{N} 27-\mathrm{C} 28$ & $1.406(3)$ & $\mathrm{C} 30-\mathrm{H} 30 \mathrm{~A}$ & 0.9800 \\
\hline $\mathrm{N} 27-\mathrm{C} 26$ & $1.396(3)$ & $\mathrm{C} 30-\mathrm{H} 30 \mathrm{~B}$ & 0.9800 \\
\hline $\mathrm{N} 27-\mathrm{C} 33$ & $1.481(3)$ & $\mathrm{C} 30-\mathrm{H} 30 \mathrm{C}$ & 0.9800 \\
\hline $\mathrm{N} 41-\mathrm{C} 42$ & $1.339(3)$ & C71-H71A & 0.9800 \\
\hline $\mathrm{N} 41-\mathrm{C} 49$ & $1.387(3)$ & C71-H71B & 0.9800 \\
\hline $\mathrm{N} 41-\mathrm{C} 50$ & $1.458(3)$ & $\mathrm{C} 71-\mathrm{H} 71 \mathrm{C}$ & 0.9800 \\
\hline $\mathrm{N} 23-\mathrm{C} 22$ & $1.339(3)$ & $\mathrm{C} 31-\mathrm{H} 31 \mathrm{~A}$ & 0.9800 \\
\hline $\mathrm{N} 23-\mathrm{C} 24$ & $1.358(3)$ & C31-H31B & 0.9800 \\
\hline N67-C68 & $1.403(3)$ & $\mathrm{C} 31-\mathrm{H} 31 \mathrm{C}$ & 0.9800 \\
\hline N67-C66 & $1.399(3)$ & $\mathrm{C} 51-\mathrm{H} 51 \mathrm{~A}$ & 0.9800 \\
\hline N67-C73 & $1.467(3)$ & C51-H51B & 0.9800 \\
\hline $\mathrm{N} 21-\mathrm{C} 22$ & $1.342(3)$ & $\mathrm{C} 51-\mathrm{H} 51 \mathrm{C}$ & 0.9800 \\
\hline $\mathrm{N} 21-\mathrm{C} 29$ & $1.385(3)$ & $\mathrm{C} 50-\mathrm{H} 50 \mathrm{~A}$ & $0.94(3)$ \\
\hline $\mathrm{N} 21-\mathrm{C} 30$ & $1.456(3)$ & $\mathrm{C} 50-\mathrm{H} 50 \mathrm{~B}$ & $0.96(4)$ \\
\hline N61-C62 & $1.336(3)$ & $\mathrm{C} 50-\mathrm{H} 50 \mathrm{C}$ & $1.00(4)$ \\
\hline N61-C69 & $1.385(3)$ & $\mathrm{C} 33-\mathrm{H} 33 \mathrm{~A}$ & 0.9800 \\
\hline N61-C70 & $1.461(3)$ & $\mathrm{C} 33-\mathrm{H} 33 \mathrm{~B}$ & 0.9800 \\
\hline N63-C64 & $1.353(3)$ & $\mathrm{C} 33-\mathrm{H} 33 \mathrm{C}$ & 0.9800 \\
\hline N63-C62 & $1.338(3)$ & $\mathrm{C} 53-\mathrm{H} 53 \mathrm{~A}$ & 0.9800 \\
\hline $\mathrm{O} 103-\mathrm{H} 10 \mathrm{~K}$ & $0.78(5)$ & C53-H53B & 0.9800 \\
\hline O103-H10L & $0.84(5)$ & $\mathrm{C} 53-\mathrm{H} 53 \mathrm{C}$ & 0.9800 \\
\hline $\mathrm{N} 45-\mathrm{C} 46$ & $1.374(3)$ & $\mathrm{C} 73-\mathrm{H} 73 \mathrm{~A}$ & 0.9800 \\
\hline $\mathrm{N} 45-\mathrm{C} 44$ & $1.375(3)$ & C73-H73B & 0.9800 \\
\hline $\mathrm{N} 45-\mathrm{C} 51$ & $1.466(3)$ & $\mathrm{C} 73-\mathrm{H} 73 \mathrm{C}$ & 0.9800 \\
\hline $\mathrm{N} 47-\mathrm{C} 48$ & $1.412(3)$ & $\mathrm{C} 70-\mathrm{H} 70 \mathrm{~A}$ & 0.9800 \\
\hline $\mathrm{N} 47-\mathrm{C} 46$ & $1.396(3)$ & $\mathrm{C} 70-\mathrm{H} 70 \mathrm{~B}$ & 0.9800 \\
\hline $\mathrm{N} 47-\mathrm{C} 53$ & $1.471(3)$ & $\mathrm{C} 70-\mathrm{H} 70 \mathrm{C}$ & 0.9800 \\
\hline $\mathrm{N} 43-\mathrm{C} 42$ & $1.336(3)$ & & \\
\hline $\mathrm{C} 8-\mathrm{O} 12-\mathrm{H} 12$ & $107(2)$ & $\mathrm{N} 23-\mathrm{C} 24-\mathrm{C} 29$ & $112.27(18)$ \\
\hline $\mathrm{C} 6-\mathrm{O} 10-\mathrm{H} 10$ & $108(3)$ & $\mathrm{C} 29-\mathrm{C} 24-\mathrm{N} 25$ & $122.19(18)$ \\
\hline
\end{tabular}




\begin{tabular}{|c|c|c|c|}
\hline $\mathrm{H} 10 \mathrm{~A}-\mathrm{O} 102-\mathrm{H} 10 \mathrm{~B}$ & $109(3)$ & $\mathrm{O} 12-\mathrm{C} 8-\mathrm{C} 9$ & $123.47(18)$ \\
\hline $\mathrm{C} 7-\mathrm{O} 11-\mathrm{H} 11$ & $111(2)$ & $\mathrm{O} 12-\mathrm{C} 8-\mathrm{C} 7$ & $116.40(18)$ \\
\hline $\mathrm{C} 3-\mathrm{O} 1-\mathrm{H} 1$ & $111(2)$ & $\mathrm{C} 9-\mathrm{C} 8-\mathrm{C} 7$ & $120.14(18)$ \\
\hline $\mathrm{H} 10 \mathrm{C}-\mathrm{O} 100-\mathrm{H} 10 \mathrm{D}$ & $97(3)$ & N61-C69-C68 & $132.3(2)$ \\
\hline $\mathrm{H} 10 \mathrm{E}-\mathrm{O} 105-\mathrm{H} 10 \mathrm{~F}$ & $109(4)$ & $\mathrm{C} 64-\mathrm{C} 69-\mathrm{N} 61$ & $104.82(17)$ \\
\hline $\mathrm{H} 10 \mathrm{G}-\mathrm{O} 104-\mathrm{H} 10 \mathrm{H}$ & $105(3)$ & C64-C69-C68 & $122.86(19)$ \\
\hline $\mathrm{H} 10 \mathrm{I}-\mathrm{O} 101-\mathrm{H} 10 \mathrm{~J}$ & $99(3)$ & $\mathrm{O} 11-\mathrm{C} 7-\mathrm{C} 6$ & $121.96(18)$ \\
\hline $\mathrm{C} 28-\mathrm{N} 27-\mathrm{C} 33$ & $116.29(17)$ & $\mathrm{O} 11-\mathrm{C} 7-\mathrm{C} 8$ & $118.61(18)$ \\
\hline $\mathrm{C} 26-\mathrm{N} 27-\mathrm{C} 28$ & $126.45(17)$ & $\mathrm{C} 8-\mathrm{C} 7-\mathrm{C} 6$ & $119.43(18)$ \\
\hline $\mathrm{C} 26-\mathrm{N} 27-\mathrm{C} 33$ & $117.24(17)$ & $\mathrm{N} 41-\mathrm{C} 49-\mathrm{C} 48$ & $131.5(2)$ \\
\hline $\mathrm{C} 42-\mathrm{N} 41-\mathrm{C} 49$ & $106.10(18)$ & $\mathrm{C} 44-\mathrm{C} 49-\mathrm{N} 41$ & $105.08(18)$ \\
\hline $\mathrm{C} 42-\mathrm{N} 41-\mathrm{C} 50$ & $126.3(2)$ & $\mathrm{C} 44-\mathrm{C} 49-\mathrm{C} 48$ & $123.34(19)$ \\
\hline $\mathrm{C} 49-\mathrm{N} 41-\mathrm{C} 50$ & $127.5(2)$ & $\mathrm{O} 54-\mathrm{C} 48-\mathrm{N} 47$ & $121.84(19)$ \\
\hline $\mathrm{C} 22-\mathrm{N} 23-\mathrm{C} 24$ & $103.11(17)$ & $\mathrm{O} 54-\mathrm{C} 48-\mathrm{C} 49$ & $127.2(2)$ \\
\hline C68-N67-C73 & $117.64(18)$ & $\mathrm{N} 47-\mathrm{C} 48-\mathrm{C} 49$ & 110.97 (19) \\
\hline C66-N67-C68 & $126.75(17)$ & $\mathrm{O} 52-\mathrm{C} 46-\mathrm{N} 45$ & $121.3(2)$ \\
\hline $\mathrm{C} 66-\mathrm{N} 67-\mathrm{C} 73$ & $115.61(18)$ & $\mathrm{O} 52-\mathrm{C} 46-\mathrm{N} 47$ & $121.2(2)$ \\
\hline $\mathrm{C} 22-\mathrm{N} 21-\mathrm{C} 29$ & $106.10(17)$ & $\mathrm{N} 45-\mathrm{C} 46-\mathrm{N} 47$ & $117.47(18)$ \\
\hline $\mathrm{C} 22-\mathrm{N} 21-\mathrm{C} 30$ & $126.55(18)$ & $\mathrm{N} 21-\mathrm{C} 30-\mathrm{H} 30 \mathrm{~A}$ & 109.5 \\
\hline $\mathrm{C} 29-\mathrm{N} 21-\mathrm{C} 30$ & $127.34(17)$ & $\mathrm{N} 21-\mathrm{C} 30-\mathrm{H} 30 \mathrm{~B}$ & 109.5 \\
\hline C62-N61-C69 & $106.06(18)$ & $\mathrm{N} 21-\mathrm{C} 30-\mathrm{H} 30 \mathrm{C}$ & 109.5 \\
\hline $\mathrm{C} 62-\mathrm{N} 61-\mathrm{C} 70$ & 126.25 (19) & $\mathrm{H} 30 \mathrm{~A}-\mathrm{C} 30-\mathrm{H} 30 \mathrm{~B}$ & 109.5 \\
\hline C69-N61-C70 & $127.66(18)$ & $\mathrm{H} 30 \mathrm{~A}-\mathrm{C} 30-\mathrm{H} 30 \mathrm{C}$ & 109.5 \\
\hline C62-N63-C64 & $103.07(18)$ & $\mathrm{H} 30 \mathrm{~B}-\mathrm{C} 30-\mathrm{H} 30 \mathrm{C}$ & 109.5 \\
\hline $\mathrm{H} 10 \mathrm{~K}-\mathrm{O} 103-\mathrm{H} 10 \mathrm{~L}$ & $110(5)$ & $\mathrm{N} 43-\mathrm{C} 44-\mathrm{N} 45$ & $126.21(19)$ \\
\hline $\mathrm{C} 46-\mathrm{N} 45-\mathrm{C} 44$ & $119.25(18)$ & $\mathrm{N} 43-\mathrm{C} 44-\mathrm{C} 49$ & $111.87(18)$ \\
\hline $\mathrm{C} 46-\mathrm{N} 45-\mathrm{C} 51$ & $119.00(18)$ & $\mathrm{C} 49-\mathrm{C} 44-\mathrm{N} 45$ & $121.92(18)$ \\
\hline $\mathrm{C} 44-\mathrm{N} 45-\mathrm{C} 51$ & $121.74(18)$ & $\mathrm{O} 72-\mathrm{C} 66-\mathrm{N} 67$ & $121.06(19)$ \\
\hline $\mathrm{C} 48-\mathrm{N} 47-\mathrm{C} 53$ & 117.03 (19) & $\mathrm{O} 72-\mathrm{C} 66-\mathrm{N} 65$ & $121.9(2)$ \\
\hline $\mathrm{C} 46-\mathrm{N} 47-\mathrm{C} 48$ & $127.02(18)$ & N65-C66-N67 & $117.06(18)$ \\
\hline $\mathrm{C} 46-\mathrm{N} 47-\mathrm{C} 53$ & $115.92(18)$ & N65-C71-H71A & 109.5 \\
\hline $\mathrm{C} 42-\mathrm{N} 43-\mathrm{C} 44$ & $103.33(18)$ & N65-C71-H71B & 109.5 \\
\hline $\mathrm{C} 26-\mathrm{N} 25-\mathrm{C} 24$ & $119.03(17)$ & $\mathrm{N} 65-\mathrm{C} 71-\mathrm{H} 71 \mathrm{C}$ & 109.5 \\
\hline $\mathrm{C} 26-\mathrm{N} 25-\mathrm{C} 31$ & $120.56(18)$ & $\mathrm{H} 71 \mathrm{~A}-\mathrm{C} 71-\mathrm{H} 71 \mathrm{~B}$ & 109.5 \\
\hline $\mathrm{C} 24-\mathrm{N} 25-\mathrm{C} 31$ & $120.40(17)$ & $\mathrm{H} 71 \mathrm{~A}-\mathrm{C} 71-\mathrm{H} 71 \mathrm{C}$ & 109.5 \\
\hline C64-N65-C66 & $119.72(17)$ & $\mathrm{H} 71 \mathrm{~B}-\mathrm{C} 71-\mathrm{H} 71 \mathrm{C}$ & 109.5 \\
\hline C64-N65-C71 & $120.76(17)$ & $\mathrm{N} 25-\mathrm{C} 31-\mathrm{H} 31 \mathrm{~A}$ & 109.5 \\
\hline C66-N65-C71 & $119.51(18)$ & $\mathrm{N} 25-\mathrm{C} 31-\mathrm{H} 31 \mathrm{~B}$ & 109.5 \\
\hline $\mathrm{C} 4-\mathrm{C} 9-\mathrm{H} 9$ & $122.5(15)$ & $\mathrm{N} 25-\mathrm{C} 31-\mathrm{H} 31 \mathrm{C}$ & 109.5 \\
\hline $\mathrm{C} 8-\mathrm{C} 9-\mathrm{C} 4$ & $119.88(18)$ & $\mathrm{H} 31 \mathrm{~A}-\mathrm{C} 31-\mathrm{H} 31 \mathrm{~B}$ & 109.5 \\
\hline $\mathrm{C} 8-\mathrm{C} 9-\mathrm{H} 9$ & $117.6(15)$ & $\mathrm{H} 31 \mathrm{~A}-\mathrm{C} 31-\mathrm{H} 31 \mathrm{C}$ & 109.5 \\
\hline $\mathrm{O} 34-\mathrm{C} 28-\mathrm{N} 27$ & $120.75(18)$ & $\mathrm{H} 31 \mathrm{~B}-\mathrm{C} 31-\mathrm{H} 31 \mathrm{C}$ & 109.5 \\
\hline $\mathrm{O} 34-\mathrm{C} 28-\mathrm{C} 29$ & $127.67(19)$ & $\mathrm{N} 45-\mathrm{C} 51-\mathrm{H} 51 \mathrm{~A}$ & 109.5 \\
\hline $\mathrm{N} 27-\mathrm{C} 28-\mathrm{C} 29$ & $111.58(17)$ & $\mathrm{N} 45-\mathrm{C} 51-\mathrm{H} 51 \mathrm{~B}$ & 109.5 \\
\hline $\mathrm{O} 74-\mathrm{C} 68-\mathrm{N} 67$ & $121.51(19)$ & $\mathrm{N} 45-\mathrm{C} 51-\mathrm{H} 51 \mathrm{C}$ & 109.5 \\
\hline $\mathrm{O} 74-\mathrm{C} 68-\mathrm{C} 69$ & $126.7(2)$ & $\mathrm{H} 51 \mathrm{~A}-\mathrm{C} 51-\mathrm{H} 51 \mathrm{~B}$ & 109.5 \\
\hline N67-C68-C69 & $111.75(18)$ & $\mathrm{H} 51 \mathrm{~A}-\mathrm{C} 51-\mathrm{H} 51 \mathrm{C}$ & 109.5 \\
\hline $\mathrm{N} 63-\mathrm{C} 64-\mathrm{N} 65$ & $126.15(19)$ & $\mathrm{H} 51 \mathrm{~B}-\mathrm{C} 51-\mathrm{H} 51 \mathrm{C}$ & 109.5 \\
\hline
\end{tabular}




$\begin{array}{ll}\mathrm{N} 63-\mathrm{C} 64-\mathrm{C} 69 & 112.08(18) \\ \mathrm{N} 65-\mathrm{C} 64-\mathrm{C} 69 & 121.77(18) \\ \mathrm{C} 4-\mathrm{C} 5-\mathrm{H} 5 & 122.4(16) \\ \mathrm{C} 6-\mathrm{C} 5-\mathrm{C} 4 & 119.37(19) \\ \mathrm{C} 6-\mathrm{C} 5-\mathrm{H} 5 & 118.2(16) \\ \mathrm{N} 23-\mathrm{C} 22-\mathrm{N} 21 & 113.42(19) \\ \mathrm{N} 23-\mathrm{C} 22-\mathrm{H} 22 & 126.9(17) \\ \mathrm{N} 21-\mathrm{C} 22-\mathrm{H} 22 & 119.4(17) \\ \mathrm{C} 9-\mathrm{C} 4-\mathrm{C} 3 & 121.08(18) \\ \mathrm{C} 5-\mathrm{C} 4-\mathrm{C} 9 & 120.66(18) \\ \mathrm{C} 5-\mathrm{C} 4-\mathrm{C} 3 & 118.26(18) \\ \mathrm{O} 32-\mathrm{C} 26-\mathrm{N} 27 & 120.64(19) \\ \mathrm{O} 32-\mathrm{C} 26-\mathrm{N} 25 & 121.57(19) \\ \mathrm{N} 25-\mathrm{C} 26-\mathrm{N} 27 & 117.80(18) \\ \mathrm{O} 1-\mathrm{C} 3-\mathrm{C} 4 & 112.93(18) \\ \mathrm{O} 2-\mathrm{C} 3-\mathrm{O} 1 & 122.85(19) \\ \mathrm{O} 2-\mathrm{C} 3-\mathrm{C} 4 & 124.22(19) \\ \mathrm{N} 41-\mathrm{C} 42-\mathrm{H} 42 & 123(2) \\ \mathrm{N} 43-\mathrm{C} 42-\mathrm{N} 41 & 113.62(19) \\ \mathrm{N} 43-\mathrm{C} 42-\mathrm{H} 42 & 123(2) \\ \mathrm{N} 61-\mathrm{C} 62-\mathrm{N} 63 & 113.97(19) \\ \mathrm{N} 61-\mathrm{C} 62-\mathrm{H} 62 & 122.8(19) \\ \mathrm{N} 63-\mathrm{C} 62-\mathrm{H} 62 & 123.2(19) \\ \mathrm{O} 10-\mathrm{C} 6-\mathrm{C} 5 & 123.93(18) \\ \mathrm{O} 10-\mathrm{C} 6-\mathrm{C} 7 & 115.58(18) \\ \mathrm{C} 5-\mathrm{C} 6-\mathrm{C} 7 & 120.49(19) \\ \mathrm{N} 21-\mathrm{C} 29-\mathrm{C} 28 & 131.99(19) \\ \mathrm{C} 24-\mathrm{C} 29-\mathrm{N} 21 & 105.09(17) \\ \mathrm{C} 24-\mathrm{C} 29-\mathrm{C} 28 & 122.92(18) \\ \mathrm{N} 23-\mathrm{C} 24-\mathrm{N} 25 & 125.54(18) \\ & \end{array}$

$\begin{array}{ll}\text { N41-C50-H50A } & 108(2) \\ \text { N41-C50-H50B } & 107(3) \\ \text { N41-C50-H50C } & 108(2) \\ \text { H50A-C50-H50B } & 107(3) \\ \text { H50A-C50-H50C } & 115(3) \\ \text { H50B-C50-H50C } & 111(3) \\ \text { N27-C 33-H33A } & 109.5 \\ \text { N27-C 33-H33B } & 109.5 \\ \text { N27-C33-H33C } & 109.5 \\ \text { H33A-C33-H33B } & 109.5 \\ \text { H33A-C33-H33C } & 109.5 \\ \text { H33B-C33-H33C } & 109.5 \\ \text { N47-C53-H53A } & 109.5 \\ \text { N47-C53-H53B } & 109.5 \\ \text { N47-C53-H53C } & 109.5 \\ \text { H53A-C53-H53B } & 109.5 \\ \text { H53A-C53-H53C } & 109.5 \\ \text { H53B-C53-H53C } & 109.5 \\ \text { N67-C73-H73A } & 109.5 \\ \text { N67-C73-H73B } & 109.5 \\ \text { N67-C73-H73C } & 109.5 \\ \text { H73A-C73-H73B } & 109.5 \\ \text { H73A-C73-H73C } & 109.5 \\ \text { H73B-C73-H73C } & 109.5 \\ \text { N61-C70-H70A } & 109.5 \\ \text { N61-C70-H70B } & 109.5 \\ \text { N61-C70-H70C } & 109.5 \\ \text { H70A-C70-H70B } & 109.5 \\ \text { H70A-C70-H70C } & 109.5 \\ \text { H70B-C70-H70C } & 109.5\end{array}$

Hydrogen-bond geometry $\left(A,{ }^{\circ}\right)$

\begin{tabular}{lllll}
\hline$D-\mathrm{H} \cdots A$ & $D-\mathrm{H}$ & $\mathrm{H} \cdots A$ & $D \cdots A$ & $D-\mathrm{H}^{\cdots} A$ \\
\hline $\mathrm{O} 1-\mathrm{H} 1 \cdots \mathrm{O} 32^{\mathrm{i}}$ & $0.85(3)$ & $1.86(3)$ & $2.672(2)$ & $161(3)$ \\
$\mathrm{O} 10-\mathrm{H} 10 \cdots \mathrm{O} 104$ & $0.86(4)$ & $1.79(4)$ & $2.643(2)$ & $174(4)$ \\
$\mathrm{O} 11-\mathrm{H} 11 \cdots \mathrm{O} 10$ & $0.83(3)$ & $2.32(4)$ & $2.709(2)$ & $109(3)$ \\
$\mathrm{O} 11-\mathrm{H} 11 \cdots \mathrm{O} 102$ & $0.83(4)$ & $1.88(4)$ & $2.680(2)$ & $161(3)$ \\
$\mathrm{O} 12-\mathrm{H} 12 \cdots \mathrm{O} 100^{\mathrm{i}}$ & $0.86(3)$ & $1.86(3)$ & $2.702(2)$ & $166(3)$ \\
$\mathrm{O} 100-\mathrm{H} 10 C \cdots \mathrm{O} 105$ & $0.83(4)$ & $2.07(4)$ & $2.851(3)$ & $157(4)$ \\
$\mathrm{O} 100-\mathrm{H} 10 D^{\cdots} \mathrm{O} 10$ & $0.82(4)$ & $2.74(3)$ & $3.286(2)$ & $126(3)$ \\
$\mathrm{O} 100-\mathrm{H} 10 D^{\cdots} \mathrm{O} 102$ & $0.82(4)$ & $2.02(4)$ & $2.798(3)$ & $158(3)$ \\
$\mathrm{O} 101-\mathrm{H} 10 I^{\cdots} \mathrm{N} 43^{\mathrm{ii}}$ & $0.94(4)$ & $1.97(4)$ & $2.898(3)$ & $170(3)$ \\
$\mathrm{O} 101-\mathrm{H} 10 J \cdots \mathrm{O} 100$ & $0.88(4)$ & $2.09(4)$ & $2.960(3)$ & $170(4)$ \\
$\mathrm{O} 102-\mathrm{H} 10 A \cdots \mathrm{O} 101^{\mathrm{iii}}$ & $0.89(4)$ & $1.87(4)$ & $2.754(3)$ & $169(4)$ \\
$\mathrm{O} 102-\mathrm{H} 10 B \cdots \mathrm{O} 103$ & $0.85(4)$ & $1.85(4)$ & $2.691(3)$ & $173(3)$ \\
$\mathrm{O} 103-\mathrm{H} 10 K^{\cdots} \cdots 4^{\mathrm{iv}}$ & $0.78(5)$ & $2.06(5)$ & $2.832(3)$ & $168(5)$ \\
$\mathrm{O} 103-\mathrm{H} 10 L \cdots \mathrm{N} 23^{\mathrm{v}}$ & $0.84(5)$ & $1.99(5)$ & $2.823(3)$ & $168(4)$
\end{tabular}


supporting information

$\begin{array}{lllll}\mathrm{O} 104-\mathrm{H} 10 G \cdots \mathrm{O} 1^{\text {vi }} & 0.91(3) & 2.50(3) & 3.011(2) & 116(2) \\ \mathrm{O} 104-\mathrm{H} 10 G \cdots \mathrm{O} 105 & 0.91(3) & 2.03(3) & 2.857(3) & 151(3) \\ \mathrm{O} 104-\mathrm{H} 10 H \cdots \mathrm{O} 74^{\mathrm{vi}} & 0.93(5) & 2.00(5) & 2.924(2) & 171(4) \\ \mathrm{O} 105-\mathrm{H} 10 E^{\cdots} \cdots 7^{\text {ii }} & 0.86(5) & 2.11(5) & 2.952(2) & 169(4) \\ \mathrm{O} 105-\mathrm{H} 10 F \cdots \mathrm{N} 63 & 0.88(4) & 1.92(4) & 2.798(2) & 173(4) \\ \mathrm{C} 22-\mathrm{H} 22 \cdots \mathrm{O} 72^{\text {vii }} & 0.97(3) & 2.38(3) & 3.227(3) & 146(2) \\ \mathrm{C} 30-\mathrm{H} 30 C \cdots \mathrm{O} 12^{\text {vii }} & 0.98 & 2.71 & 3.247(3) & 115 \\ \mathrm{C} 31-\mathrm{H} 31 C \cdots \mathrm{O} 2^{\text {vi }} & 0.98 & 2.40 & 3.326(3) & 158 \\ \mathrm{C} 42-\mathrm{H} 42 \cdots \mathrm{O} 12^{\text {viii }} & 0.92(3) & 2.45(3) & 3.254(3) & 146(3) \\ \mathrm{C} 42-\mathrm{H} 42 \cdots \mathrm{O} 72 & 0.92(3) & 2.66(3) & 3.136(3) & 113(2) \\ \mathrm{C} 62-\mathrm{H} 62 \cdots \mathrm{O} 52^{\text {vii }} & 0.96(3) & 2.24(3) & 3.119(3) & 151(3)\end{array}$

Symmetry codes: (i) $x,-y+1, z-1 / 2$; (ii) $x,-y+2, z+1 / 2$; (iii) $x, y-1, z$; (iv) $x+1,-y+1, z+1 / 2$; (v) $x+1, y, z$; (vi) $x,-y+1, z+1 / 2$; (vii) $x-1, y, z$; (viii) $x, y+1$, $z$. 